\title{
Flow in complex domains simulated by Dissipative Particle Dynamics driven by geometry-specific body-forces
}

\author{
Alireza Yazdani ${ }^{1}$ \\ Division of Applied Mathematics, Brown University, Providence, RI 02912 USA \\ Mingge Deng ${ }^{1}$ \\ Division of Applied Mathematics, Brown University, Providence, RI 02912 USA \\ Bruce Caswell \\ School of Engineering, Brown University, Providence, RI 02912 USA \\ George Em Karniadakis \\ Division of Applied Mathematics, Brown University, Providence, RI 02912 USA
}

\begin{abstract}
We demonstrate how the quality of simulations by Dissipative Particle Dynamics (DPD) of flows in complex geometries is greatly enhanced when driven by body forces suitably tailored to the geometry. In practice, the body force fields are most conveniently chosen to be the pressure gradient of the corresponding Navier-Stokes (N-S) flow. In the first of three examples, the driving-force required to yield a stagnation-point flow is derived from the pressure field of the potential flow for a lattice of counter-rotating line vortices. Such a lattice contains periodic squares bounded by streamlines with four vortices within them. Hence, the DPD simulation can be performed with periodic boundary conditions to demonstrate the value of a non-uniform driving-force without the need to model real boundaries. The second example is an irregular geometry consisting of a $2 \mathrm{D}$ rectangular cavity on one side of an otherwise uniform channel. The Navier-Stokes pressure field for the same geometry is obtained numerically, and its interpolated gradient is then employed as the driving-force for the DPD simulation. Finally, we present
\end{abstract}

\footnotetext{
${ }^{1}$ The authors contributed equally to this work
} 
a third example, where the proposed method is applied to a complex 3D geometry of an asymmetric constriction. It is shown that in each case the DPD simulations closely reproduce the Navier-Stokes solutions. Convergence rates are found to be much superior to alternative methods; in addition, the range of convergence with respect to Reynolds number and Mach number is greatly extended.

Keywords: particle methods, pressure-driven flows, stagnation-type flows, DPD, mesoscopic simulations, 3D simulations

\section{Introduction}

Fluid flows simulated by Dissipative Particle Dynamics (DPD) have been successfully demonstrated for several geometries. However, efficient convergence starting from rest is achievable mainly in regular geometries, such as rectilinear channel flow driven by a spatially uniform body force, essentially the gravity force of arbitrary strength applied to each DPD particle. Examples of successful DPD simulations of this kind include: flow in small tubes of a blood suspension of multi-scale model red cells ([1]), flow past a sphere fixed on the axis of a tube by Chen et al. ([2]), and flow of a DNA suspension in a micro-channel with rectangular side cavities by Fan et al. ([3]). However, in a study of blood flowing through a Y-junction, ([4]), convergence is not always possible. A continuum solution of such flows is achievable with equal ease whether with a body force driver or by specification of normal tractions across the channel section. Generally, a continuum flow is driven by prescription on its boundary of some combination of velocities and tractions; it may also be driven by body forces. Tractions on boundaries are a consequence of the divergence theorem, which permits the net force due to stress at interior points to be transformed onto the boundary. The equivalent operation does not exist for particle based methods. Furthermore, for particle methods inflow/outflow specifications for channel flows, so easily carried out with continuum methods, do not converge nearly as well as with body-force drivers. Random fluctuations, a crucial feature of DPD, impel particle motion in all directions. Hence, the hypothesis to be tested here is whether a body force field, derived by continuum methods in the same domain, can serve to guide the particles in the general direction of the motion defined by the boundaries.

The goal of this work is to investigate whether body forces can be tailored to guide DPD particles in complex geometries. In principle, any body force can be included in the equation of motion of the DPD particles. However, it is not immediately obvious how to specify such force fields so as to enhance the performance 
of DPD simulations of complex fluids. In this work we show with examples that a useful body-force candidate is the pressure gradient of the Navier-Stokes (N-S) flow, which can be generated analytically from known solutions or numerically from a large number of readily available CFD codes. This is a direct generalization of the uniform body force widely used to drive rectilinear DPD channel flows. Hence, the hypothesis to be tested is whether the N-S pressure gradient, derived by continuum methods in the same domain, can serve to guide the DPD particles in the general direction of the motion defined by the boundaries, and thereby achieve enhanced performance. This choice of the body force may not be appropriate to every flow. For example, in unsteady flows bounded by moving surfaces the boundary motions in both the continuum and in the DPD domains must coincide for all times. This appears to exclude free surfaces and interfaces whose positions are included in the list of unknowns.

We propose that the required body force potential is taken as the pressure field of the corresponding N-S flow governed by,

$$
\partial \mathbf{V} / \partial t+\mathbf{V} \cdot \nabla \mathbf{V}-(\mu / \rho) \nabla^{2} \mathbf{V}=-\nabla(P / \rho+\chi)
$$

where the viscosity $\mu$ and the density $\rho$ are constants. This corresponds to the typical conditions of DPD flow simulations, although compressibility effects may appear where large pressure gradients exist. The curl of the Navier-Stokes equation (1) eliminates the Bernoullian, $1 / 2 V^{2}+P / \rho+\chi$, and yields the vorticity equation in terms of the velocity field, and its gradients. For interior flows with velocity boundary conditions the velocity field is governed by the vorticity equation, and therefore remains unchanged for any choice of the scalar potential $\chi$ on the right-hand side of equation (1). If required, with the velocity field in hand, the scalar potential $P / \rho+\chi$ can found by integration of equation (1). Usually, it is determined as the single variable $P / \rho$, and when $\chi$ is the gravitational potential it is said to be absorbed into the 'modified' scalar pressure. In experimental fluid mechanics the fluid pressure is obtained by subtraction of the gravitational potential from the measured modified pressure.

Let a homogeneous Non-Newtonian fluid have extra stress $\mathbf{T}$, given by a constitutive equation expressible in terms of the velocity field. Its equation of motion is then,

$$
\partial \mathbf{V} / \partial t+\mathbf{V} \cdot \nabla \mathbf{V}-\nabla \cdot \mathbf{T}=-\nabla\left(P / \rho+P_{N S} / \rho\right)
$$

where $P_{N S}$ is the N-S pressure field derived from the solution of equation (1), and whose gradient functions as the body force in equation (2). The continuum al- 
gorithms for solving Non-Newtonian flows generally proceed by iteration starting from the linearized N-S equation. The Newtonian pressure gradient imposed as a body force is not usually a feature of such algorithms whose primitive variables are $\mathbf{V}, P$. It is displayed in equation (2) to emphasize that the unknown $P$ is then the disturbance pressure due the Non-Newtonian character of the fluid. The term 'complex fluids' is to be understood here to describe those fluids which, in addition to exhibiting nonlinear extra-stress, become spatially inhomogeneous when subjected to flow. Typically, these are suspensions of small particles and macromolecules such as those found in bio-fluids, e.g. blood. The development of constitutive equations for such heterogeneous materials is fraught with theoretical and experimental difficulties, and in some circumstances the heterogeneous continuum description becomes invalid ([1]). The alternative is the particle description where the suspended bodies are modeled as mesoscopic entities, and for the DPD method employed in this work the solvent is likewise modeled with such particles. However, flow simulations with DPD have convergence limitations, which are usually manifested by failure of the thermostat to maintain temperature uniformity. For complex domains, convergence failure generally appears at lower flow rates than would be expected for the corresponding flows in simple geometries such as straight channels and tubes. The difficulties appear to stem from the thermal fluctuations, a crucial feature of DPD, which drive the particles in random directions. Hence, our aim here is to explore the use of body force fields as local drivers to guide the particles towards the direction of the local average motion.

Here this concept is demonstrated in the DPD simulation of flow in three irregular geometries. One consists of four counter-rotating vortices inside a periodic box bounded by streamlines with stagnation points at each corner and edge midpoint. This box is a unit cell of an infinite lattice of counter-rotating, equallyspaced line vortices whose irrotational velocity field satisfies the N-S equation, and its periodicity avoids the need to model real-wall boundaries. Furthermore, since strain rates are determined by the line-vortex strength, the uniform strain rates in the stagnation region are then prescribed, and thus satisfy the design goal of the periodic box. In the second example, the irregular geometry consists of a rectangular cavity on one side of an otherwise uniform channel. The NavierStokes pressure field for the same geometry is obtained numerically with the NEKTAR ([5]) spectral element (SEM) code, and the driving force for the DPD simulation is derived from its interpolated gradient. In the final third example, we explore the applicability of the proposed method to 3D complex geometries by looking at an asymmetric constriction in a tube representing an atherosclerotic plaque in an artery. 


\section{Potential for a lattice of line vortices}

In a number of papers ([6], [7]) Leal and co-workers have experimentally realized the classical stagnation flow in the central region of a four-roll mill; see also the recent work by Deschamps et al. [8]. Here this apparatus is idealized by four line vortices within a periodic square bounded by streamlines. This potential flow without solid boundaries satisfies the N-S equation for incompressible flow, and from it we will derive the body force needed to drive the DPD simulation of the equivalent flow. Before the complete complex potential is constructed, the regions near the vortices and near the stagnation points are reviewed. For a single line vortex and for the stagnation-point flow complex potentials are respectively,

$$
W=\phi+i \psi=-i \kappa /(2 \pi) \ln (z), W=1 / 2 \dot{\varepsilon} z^{2}, z=x+i y=r e^{i \theta},
$$

where $\kappa$ is the vortex strength measured as its circulation, and $\dot{\varepsilon}$ the stagnation stretch rate; the real and imaginary parts of $W$ are the scalar velocity potential $\phi$ and the stream function $\psi$. The complex velocity is derived from $d W / d z=U-i V$ so that $\overline{d / d z}$ corresponds to the vector operator $\nabla$, and also $\left(e^{i \theta}, i e^{i \theta}\right)$ correspond to the base vectors $\left(\mathbf{k}_{r}, \mathbf{k}_{\theta}\right)$ in cylindrical-coordinates. With the overline to indicate the complex conjugate, the body force per unit mass $\nabla \chi$ is determined by Bernoulli 's equation to be the gradient of $-\left(q^{2}\right) / 2$, and is obtained for each case from equations (3), respectively, as

$$
\begin{gathered}
q^{2}=(d W / d z) \overline{d W / d z}=(\kappa / 2 \pi r)^{2}, q^{2}=\dot{\varepsilon} r^{2}, \\
-\overline{d q^{2} / d z}=\nabla \chi=(\kappa /(2 \pi))^{2}\left(\mathbf{k}_{\mathbf{r}} / r^{3}\right), \quad \nabla \chi=-\dot{\varepsilon}^{2} r \mathbf{k}_{\mathbf{r}} .
\end{gathered}
$$

Hence, the effective body force per unit mass is radial and repulsive relative to the vortex, and radial and attractive relative to the stagnation point. In viscous flow the vortex strength can be characterized by the Reynolds number $\operatorname{Re}=\kappa \rho / \mu$. The complex potential for four vortices arranged symmetrically in a square defined by streamlines requires the construction of the potential for a square lattice of vortices. The starting point is Milne-Thomson's ([9], p374) potential for an infinite row of positive vortices equally-spaced along the real axis,

$$
W_{0}(z)=-i(\kappa / 2 \pi) \ln (\sin (\pi z / a)),
$$

where $\kappa$ is the vortex strength, $a$ is the vortex spacing along $\mathrm{x}, z$ gives the fieldpoint position relative to the reference vortex at $z=0$. The vortex positions then 
proceed to the left(-) and right(+) as: $-n a, \ldots,-2 a,-1 a, 0,+1 a,+2 a, \ldots,+n a$. Milne-Thomson's result holds for a linear row at any orientation by taking the vortex spacing $a$ to be any complex constant $A$. In what follows below, the reference origin will lie at the center of symmetry of a square, and the following definitions will be needed for the construction of the potential of the lattice of vortices,

$$
2 z=2 z_{j k}=z_{j}+z_{k}, \pi\left(z_{j}-z_{k}\right) / A=\pi 2 b / A=\pi(n+i m),
$$

where $n, m$ are real integers for a square lattice (see Appendix). The reference origin $z_{j k}$ is the midpoint between any two reference vortices $(j, k)$ separated by $z_{j}-z_{k}=2 b$ along the same row $(m=0)$, or when $j$ and $k$ lie on separate parallel rows $(m \neq 0)$ then $z_{j k}$ lies between the rows at the midpoint of $z_{j}-z_{k}$. For both cases, the construction of the total potential requires the sums of row potentials referred to the midpoint $z_{j k}$.

Let $W_{j}$ and $W_{k}$ be the Mine-Thomson vortex row potential (6) with origins at vortices $j$ and $k$ respectively, and with the real line spacing $a$ replaced by a complex spacing $A$, a vector oriented in any direction. The sum of these potentials $W_{j k}=W_{j}+W_{k}$ introduces sine products in $z_{j}$ and $z_{k}$, which must be transformed to the midpoint reference origin $z_{j k}$. This is accomplished by standard identities for products of trigonometric functions ([10], p78) as,

$$
\begin{aligned}
\sin \left(\pi z_{j} / A\right) \sin \left(\pi z_{k} / A\right) & =\sin \left(\pi\left(\left(z_{j k}-b\right) / A\right)\right) \sin \left(\pi\left(\left(z_{j k}+b\right) / A\right)\right. \\
& =\sin \left(\pi z_{j k} / A\right)^{2}-\sin (\pi b / A)^{2} .
\end{aligned}
$$

The dimensionless potential $(2 \pi / \kappa) W_{j k}\left(z_{j k}\right)$ referred to the origin $z_{j k}$ at midpoint of $z_{j}-z_{k}$ is then,

$$
W(z)=-i \ln \left(\sin \left(\pi z_{j} / A\right) \sin \left(\pi z_{k} / A\right)\right)=-i \ln \left(\sin \left(z^{*}\right)^{2}-\sin (\pi b / A)^{2}\right),
$$

where $z^{*}=\pi z / A$, and henceforth the subscript of $z_{j k}$ is dropped to indicate that the sum of every pair of vortex rows will be referred to the center of a typical lattice square as shown in Fig. 1. This is the starting point for all the potentials given below. When $(j, k)$ lie on the same row $(m=0)$ the contributions of each vortex are doubled in equation (9); accordingly, to be a term in a sum over all vortices counted once, that $W(z)$ must be weighted by half. The potential (9) and its first two derivatives are now written with the condensed notations:

$$
Z_{S}\left(z^{*}, m\right), Z_{c}\left(z^{*}, m\right)=\sin \left(z^{*}\right)^{2}+\sinh (m \pi / 2)^{2},-\cosh (m \pi / 2)^{2},
$$


here the subscripts $s$ and $c$ indicate respectively that the second term is either $\sinh (m \pi / 2)$ for $n$ even, or $-\cosh (m \pi / 2)$ for $n$ odd. Thus for $n$ even,

$$
\begin{aligned}
& W_{s}\left(z^{*}, m\right)=-i \ln \left(Z_{s}\left(z^{*}, m\right)\right),(A / \pi) \frac{d W_{s}\left(z^{*}, m\right)}{d z}=-i \frac{\sin \left(2 z^{*}\right)}{Z_{s}\left(m, z^{*}\right)}, \\
& (A / \pi)^{2} \frac{d^{2} W_{s}\left(z^{*}, m\right)}{d z^{2}}=i\left\{\frac{\sin (2 z *)^{2}}{Z_{S}\left(m, z^{*}\right)^{2}}-\frac{2 \cos \left(2 z^{*}\right)}{Z_{s}\left(m, z^{*}\right)}\right\}
\end{aligned}
$$

and for $\mathrm{n}$ odd $W_{c}$ and its derivatives are obtained from equations (11) by replacing $Z_{s}$ with $Z_{c}$. The definitions (10) are insensitive to the signs of $A, n$, and $m$, consequently so are these potentials and their derivatives. For the important case $n$ odd, $m=0$, they simplify to,

$$
\begin{gathered}
W_{c}(z *, 0)=-i \ln \cos \left(z^{*}\right), \quad(A / \pi) d W_{c}\left(z^{*}, 0\right) / d z=i \tan \left(z^{*}\right), \\
(A / \pi)^{2} d^{2} W_{c}\left(z^{*}, 0\right) / d z^{2}=i \sec \left(z^{*}\right)^{2},
\end{gathered}
$$

where the extraneous constant $\ln (-1)$ has been omitted from $W(z)$ which is normalized by $1 / 2$ to satisfy equal weighting of vortices.

Figure 1 depicts the lattice of vortices with alternating signs whose structure consists of periodic squares bounded by streamlines and containing four vortices. The vortex positions define squares of side $a$; the red circle marks the center of a typical vortex square. The periodic squares of side $2 a$ have corners, marked with open circles, at the centers of diagonally neighboring squares. The center of each square is a stagnation point near which the motion is the stagnation-point flow treated above. When all vortices have one sign a circulating flow is produced in each periodic square. The diagonals of the lattice are vortex rows of entirely one sign with intersecting right and left diagonals of the same sign passing though each vortex. Successive vortices along the diagonals are connected by the vectors:

$$
A_{r}=a+i a, A_{l}=-a+i a, A_{r} A_{l}=-2 a^{2}, A_{r} / A_{l}=-i=-A_{l} / A_{r},
$$

where the subscripts $r$ and $l$, respectively, indicate right and left vectors connecting diagonally adjacent vortices. Thus, the notations $z^{*}{ }_{r}, z^{*}{ }_{l}$ imply from its definition (10) that $z^{*}$ is to be constructed with $A_{r}$ or $A_{l}$, respectively. The complete potential for the lattice of vortices is constructed as a weighted sum of the row potentials (11) for the diagonals passing through the labeled vortices in Fig. 1. The weights are chosen so that each vortex is counted once only. The details of this construction are given in Appendix, and from the terms given there the total dimensional potential follows by induction to be, 


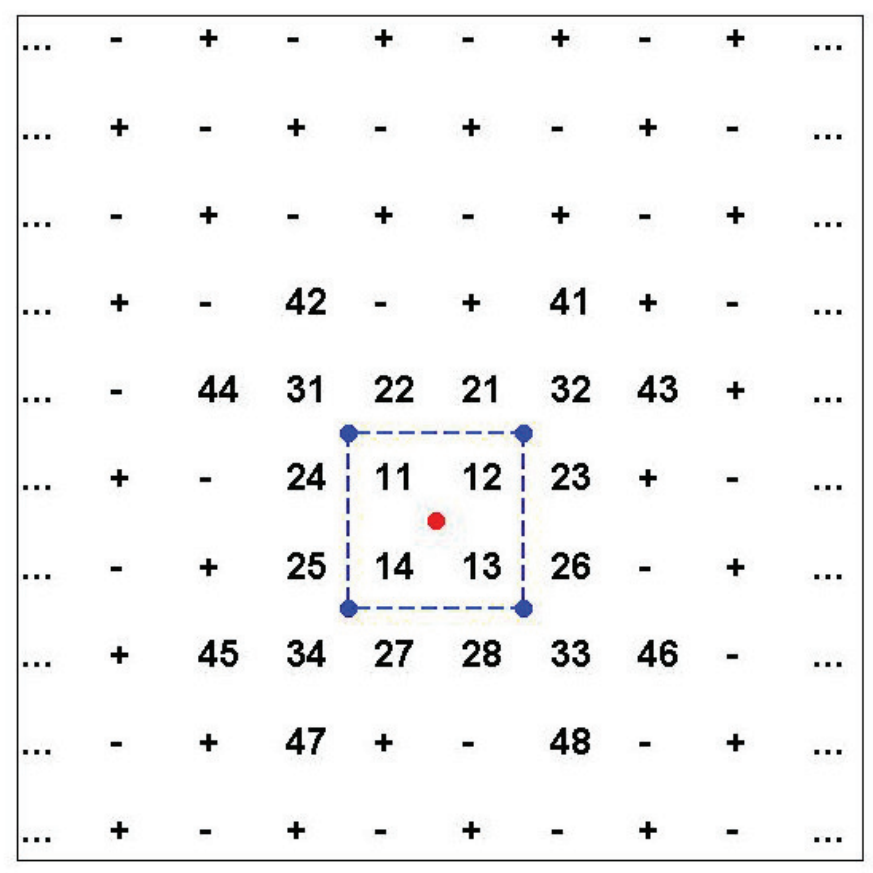

Figure 1: Lattice of vortices with alternating signs and spacing $a$. The dashed lines define a typical periodic square of side $2 \mathrm{a}$; the vortices labeled with two-digits refer to the construction of the potential, equation (14), described in Appendix. Alternating signs (shown) yield the stagnationpoint flow, and same signs (not shown) the circulation flow in each periodic square.

$$
\begin{aligned}
& W=-i \kappa /(2 \pi)\{\quad \ln \left\{\cos \left(z *_{r}\right) \prod_{k=1}^{\infty} W_{s}\left(z *_{l},(2 k-1)\right) W_{c}\left(z *_{r}, 2 k\right)\right\} \\
&\left.\mp \ln \left\{\cos \left(z *_{l}\right) \prod_{k=1}^{\infty} W_{s}\left(z *_{r},(2 k-1)\right) W_{c}\left(z *_{l}, 2 k\right)\right\}\right\}
\end{aligned}
$$

where $\mp$ indicates the stagnation-point flow and the circulation flow respectively. Shear flow is realized by elimination of the negative vortices following $\mp$. The potential is then obtained from equations (6) to (14) by omission of all terms following the $\mp$ signs from equation (14). The stagnation-point flow and the shear flow can be superposed to yield mixed stretching-shear flows in arbitrary proportions in the vicinity of the origin $z=0$. Likewise, the derivatives $d W / d z$ and $d^{2} W / d z^{2}$ can be represented as infinite sums. The infinite products in equation (14) converge rapidly so that only four or five terms are sufficient for an accurate representation of $W$ in the periodic square whose center is $z=0$. 

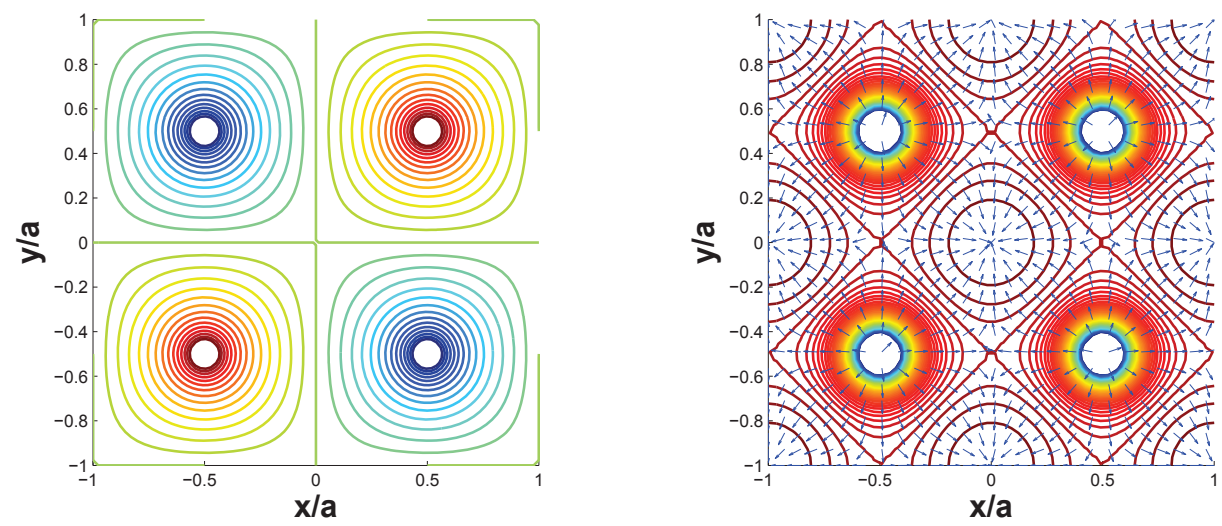

Figure 2: Periodic square of a lattice of counter-rotating vortices: (left) dimensionless stream function, and (right) pressure contours, unit arrows indicate direction of the pressure gradient field derived from equation (14). Arrows point away from vortices and towards stagnation points as suggested by equations (5).

Figure 2(left) displays streamlines derived from the imaginary part of the complex potential (14). In potential flow the Bernoullian, $H=1 / 2 \rho q^{2}+P+\rho \chi$ is constant, and hence $-1 / 2 q^{2}$ is effectively the pressure depicted in Fig. 2(right), which is derived from the derivatives of $W$, as in the limiting cases discussed above (see equation (5)). The arrows on the pressure contours are unit vectors indicating the direction of the driving body force. Due to the singularities at the vortex positions, contours plot as an almost continuous band, and hence for clarity are omitted in their vicinity. This emphasizes their circular form as expected from the well-known single vortex results summarized in equation (5). The elementary potentials and their derivatives (3), (5) suggest that a contour plot of $P / \rho$ should show concentric circles in the vicinities of the vortices and the stagnation point with arrows pointing radially outward from the vortices and inward towards the stagnation points.

In the stagnation region near $z=0$, the potential $W$ approaches the elementary stagnation-point potential $1 / 2 \dot{\varepsilon} z^{2}$. As $z \rightarrow 0$ the expansion of expression (14) yields,

$$
\begin{array}{r}
W=\left(\kappa /(2 \pi)(\pi z / a)^{2}\left\{1 / 2+\sinh (\pi / 2)^{-2}+\cosh (\pi)^{-2}+\right.\right. \\
\left.\sinh (3 \pi / 2)^{-2}+\cosh (2 \pi)^{-2}+\ldots\right\}=0.6966 \ldots\left(\kappa /(2 \pi)(\pi z / a)^{2} .\right.
\end{array}
$$

The very small contribution of the last term suggests that contributions from image vortices outside the periodic square are well-captured by this truncation of 
$W$, equation (14) at $W_{4}$ (see Appendix). From equation (3), the stagnation-point strain rate, $\dot{\varepsilon}=2.1884 \kappa / a^{2}$, is specified by the choice of the vortex strength and spacing.

\section{DPD simulation}

The DPD method, see Appendix, employed in the following examples is essentially that described in detail by Lei et al. ([11]), and in this work the "standard DPD particles" are those described therein. The significant difference being the external body force imposed on each particle $i$ at its current position $\mathbf{r}_{i}$, $\mathbf{F}_{i}^{\text {ext }}=(1 / \rho) \nabla P\left(\mathbf{r}_{i}\right)$, by interpolation of the known continuum-pressure gradient.

\subsection{Example 1: stagnation-point flow}

Standard DPD particles occupy a periodic box of size $20^{3}$. On the $x-y$ planes of the domain, the body force field is imposed, derived from the gradient of the analytical pressure field for a lattice of vortices shown in Fig. 2. Since the analytical potential flow and the periodic boundary conditions satisfy the N-S equation this system of DPD particles is expected to respond macroscopically as a Newtonian fluid. Hence, verification of the driving force hypothesis implies that the DPD results should agree closely with the analytical fields. The DPD parameters employed here are given in Table 1.

\begin{tabular}{ccccccccc}
\hline$n$ & $a_{c}$ & $a_{d}$ & $s$ & $r_{c}$ & $k_{B} T$ & $v$ & $c_{s}$ & $\operatorname{Re}_{c}$ \\
\hline 6.0 & 25.0 & 5.0 & 1.0 & 1.0 & 0.1 & 5.60 & 5.6 & 20.0 \\
\hline 6.0 & 12.5 & 5.0 & 1.0 & 1.0 & 0.1 & 5.60 & 4.0 & 14.3 \\
\hline 5.0 & 15.0 & 5.0 & 1.0 & 1.0 & 0.1 & 3.88 & 4.0 & 20.6 \\
\hline 4.0 & 18.75 & 5.0 & 1.0 & 1.0 & 0.1 & 2.49 & 4.0 & 32.1 \\
\hline 3.0 & 25.0 & 5.0 & 1.0 & 1.0 & 0.1 & 1.41 & 4.0 & 56.7 \\
\hline
\end{tabular}

Table 1: DPD parameters: $n$ number density, $a_{c}, a_{d}$ conservative, dissipative force coefficients, $s$ dissipative force distribution index, $r_{c}$ cutoff radius, $k_{B} T$ Boltzmann constant $\times$ temperature. $v$ kinematic viscosity, $c_{s}$ sound speed, $R_{c}=c L / v$ the sound speed Reynolds number. All parameters are in DPD units.

In Fig. 2 the near-vortex regions were left blank since there the $\mathbf{V}, P$ fields are singular with circular contours. Figure 3 shows the streamlines calculated from the DPD velocity fields to be very similar to their analytical counterparts, except 
in the near-vortex regions. Likewise the number density, indicated by the background color of Fig. 3, shows it to be the prescribed value of 3.0 (orange) over most of the domain. In equation (5) the pressure gradient, and hence the DPD driving force, is $\mathrm{O}\left(r^{-3}\right)$ directed away from the vortices. This implies that the near-vortex regions will suffer depletion as indicated by the background colors of the plot. However, when the density gradient is normal to the circular streamlines $\nabla \cdot(\rho \mathbf{V})=\rho \nabla \cdot \mathbf{V}=0$, and hence the single-vortex velocity field continues to satisfy the equation of motion for a compressible viscous fluid with constant viscosity. For the single-vortex flow of an isentropic compressible gas, Milne-Thomson ([9], p382) argues that within the circle of unit Mach number the motion is rigid rotation. However, in isothermal gas flow this argument does not hold; none-theless in Fig. 3 significant depletion begins with the approach to the circle of unit isothermal-Mach number surrounding the vortices.



Figure 3: DPD streamlines for the periodic box in a lattice of counter-rotating vortices calculated from the time-averaged DPD velocities. Colored background indicates number density. Prescribed average density: orange, other colors indicate depletion. Near the vortices depletion starts at about a radius of unit isothermal-Mach number.

The anti-symmetry of the streamlines of Fig. 2 implies that the $x, y$-velocity components $U, V$ along the $x, y$-axes satisfy $U(x, 0)=-V(0, y)$. Likewise, the strain-rate difference $D_{x x}-D_{y y}$ and the Newtonian stress difference $T_{x x}-T_{y y}$ are the same along those axes. Fig. 4 compares the average of $U(x, 0)$ and $-V(0, y)$ 
calculated by DPD (points) with their analytical counterparts (continuous curves) for several Reynolds numbers, $\kappa \rho / \mu$. The average DPD velocity is differentiated numerically along the axes to yield the strain rate, which is also plotted against its analytical values. Also shown (see Fig. 5) is the stress difference $T_{x x}-T_{y y}$ calculated with the virial (Kirkwood) formula from the DPD simulation compared to the analytical stress calculated as the analytical strain rate times the DPD viscosity measured in a separate shear flow simulation [12]. The plotted stresses collapse onto a single curve because $\kappa$ and $\mu$ were chosen to satisfy $\kappa \mu=$ constant to verify that the DPD model satisfies the analytical scaling. The agreement between the DPD results and the analytical solution of the N-S system is clearly good, and excellent in the vicinity of the axes.

The Reynolds numbers presented for example 1 by no means represent the full range available for accurate simulations; at some yet higher $R e$ the DPD system will fail to maintain its uniform prescribed temperature. This will manifest itself as non-uniform density throughout the domain, rather than being confined to the immediate neighborhood of the vortices as shown in Fig. 3. It should also be noted that potential flow satisfies the Stokes equation with spatially uniform pressure. Hence, at very small Re, i.e. vanishing vortex strength $\kappa$, the pressure field approaches uniformity, and the DPD driving force disappears. The lowest viable $R e$ for an accurate DPD simulation has not been investigated in this work.
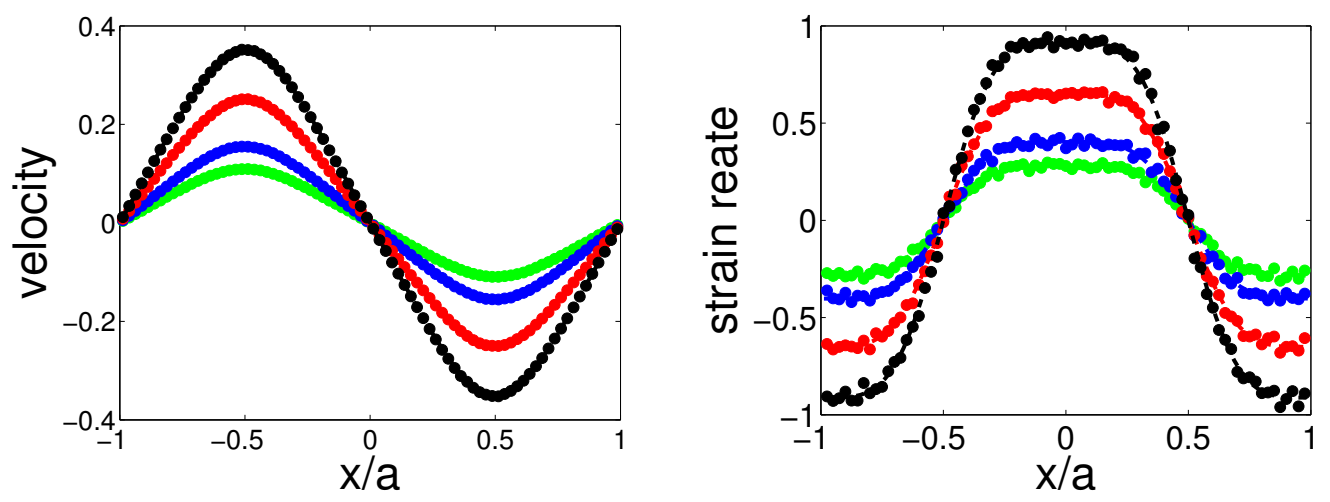

Figure 4: Periodic box of a lattice of counter-rotating vortices at Reynolds numbers 5.0 (black), 2.45 (red), 0.97 (blue), 0.45 (green):(left)dimensionless velocity components $U,-V$ and (right) strain rates $U_{x}-V_{y}$ along axes $y=0$ and $x=0$. DPD averages (points) compared to the analytical values (dashed lines) calculated from equation (15).

To study the effects of the compressibility, we first investigate the stagnationpoint flow with different speed of sound and Mach number by varying the conser- 


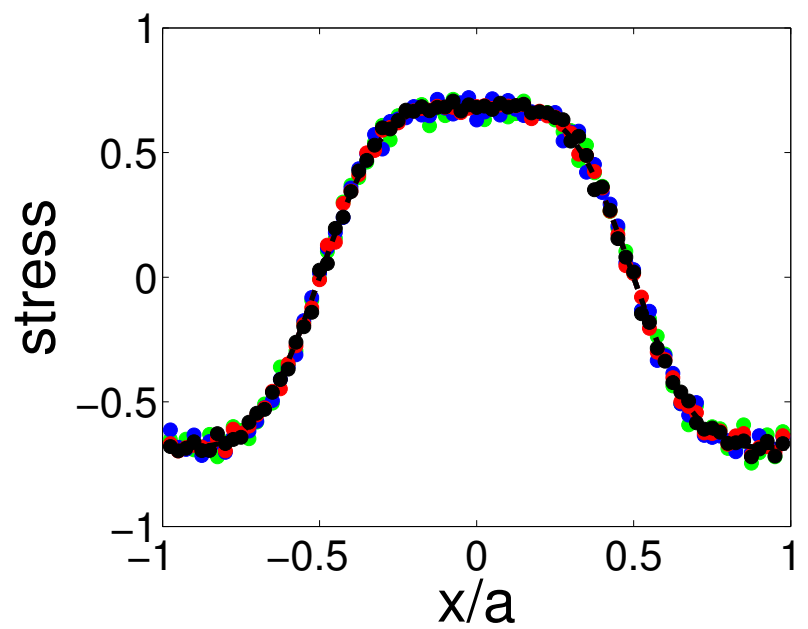

Figure 5: Periodic box of a lattice of counter-rotating vortices at Reynolds numbers of Fig. 4: dimensionless stress differences $T_{x x}-T_{y y}$ along axes $y=0$ and $x=0$. DPD averages (points) compared to values (dashed lines) calculated from strain rates derived from equation (15) multiplied by the viscosity measured in shear flow.

vative force parameter $a_{c}$ and number density $n$ shown in Table 1 . Our results in Fig. 6 show that the current methodology works very well when the Mach numbers are smaller than 1.0, whereas it shows a dramatic change and discrepancy from the desired analytical solutions when the Mach number is above 1.0. It seems that the apparent viscosity increases sharply when the Mach number is above 1.0.

The results displayed in Figs. 4, 5 suggest that the lattice of vortices is a useful device for accurately simulating the planar stagnation-point flow, and that the observed depletion is confined to a small region near the vortices. The real advantage of this realization of the stagnation-point flow driven by body forces is its use in the study of complex fluids which will be demonstrated in future work; see also [13]. The DPD simulation of uni-axial elongational flow has previously been achieved by Pan et al. ([14]) by use of an outer periodic box containing two internal parallel planes upon which uniform opposing normal velocities are imposed on particles traversing through them. We have adapted this configuration to simulate the stagnation-point flow, but have found it to have some drawbacks. Firstly, the force-driven scheme of this work converges to steady flow much faster; typically, the number of iterations is reduced by a factor of about 40. Secondly, the planes-in-a-box scheme requires a far larger number of DPD particles to achieve a satisfactory result, and thirdly, a symmetric strain rate field and its strength cannot 


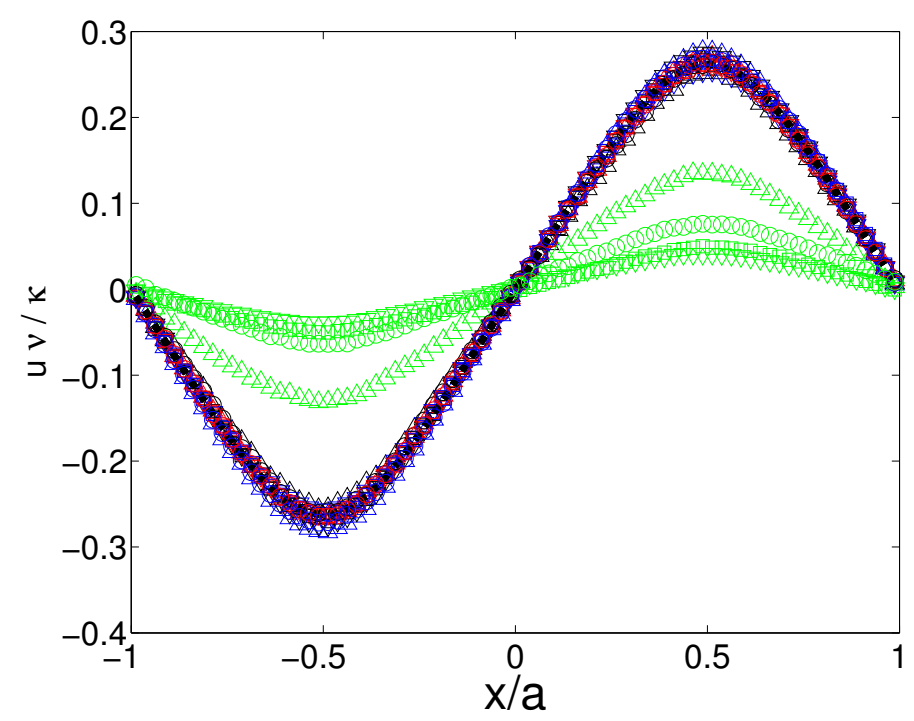

Figure 6: Dimensionless velocity rescaled by $v / \kappa$ at sound speed Reynolds numbers $\left(R e_{c}\right)$ 20.0(upper triangle), 14.3 (lower triangle), 20.6 (rectangle), 32.1(circle). All of the data perfectly collapse on analytical result (black line) when Mach number is small, the data deviate from the analytical result when Mach number is above 1.0 (green symbols).

be specified 'a priori', but must be determined by trial.

\subsection{Example 2: flow through a grooved channel}

A DPD simulation of flow through a straight plane channel or tube would normally be driven by a uniform body force directed along the channel generators. As will be demonstrated, the addition of a side cavity creates an irregular geometry which is inconsistent with such a driver. The corresponding N-S flow is solvable by numerical methods to yield a pressure field whose gradient is then used as a driver to guide DPD particles through the same irregular channel. In this example the size of cavity is relatively small which requires a higher particle density (or Lagrangian resolution). We can increase the Lagrangian resolution by either increasing the number density of the particles $n$ or through increasing the cutoff radius $r_{c}$. However, care must be taken since a larger $r_{c}$ increases the kinematic viscosity $v$ dramatically as $v \sim r_{c}^{5}$. Therefore, simulations with large values of $r_{c}$ will result in yet lower instead of higher Re. Table 2 gives the parameters for two different resolutions picked for this example. In order to sufficiently resolve the corner fields our numerical experiments revealed that at $r_{c}=1$ a density of 
$n=8$ is necessary since lower values of $n$ did not successfully capture the cavity vortex (results not shown here). We will show that increasing $r_{c}$ to 1.5 improves the accuracy, whereas the best result is achieved for resolution 1.

\begin{tabular}{lcccccccc}
\hline & $n$ & $a_{c}$ & $a_{d}$ & $s$ & $r_{c}$ & $k_{B} T$ & $v$ & $c_{s}$ \\
\hline resolution 1 & 8.0 & 9.375 & 4.5 & 0.25 & 1.0 & 1.0 & 1.11 & 4.5 \\
resolution 2 & 4.0 & 18.75 & 4.5 & 0.4 & 1.5 & 1.0 & 2.61 & 9.1
\end{tabular}

Table 2: DPD parameters: same parameters as in Table 1.

At $R e=20$, Fig. 7(a) shows the continuum pressure field in the vicinity of the cavity calculated by the continuum-based solver NEKTAR, and Fig. 7(b) shows the streamlines derived from the average velocity delivered by the DPD simulation driven by the continuum pressure gradient for resolution 1 . The boundary conditions for both types of simulation are zero velocity on the solid walls and periodic end conditions. The computational domain extends over $x=(-20,50)$ to ensure the recovery of Poiseuille flow at the exit section, and thereby justify periodicity in the streamwise direction. Only the region near the cavity is shown in the plots. Periodicity effectively extends the velocity boundary conditions on the walls to an infinitely long but irregular channel so that inflow/outflow conditions are not required.

In the vicinity of the cavity's corners Figs. 8, 9(a,b) compare profiles of the $x, y$-velocity components $U, V$ along lines of constant $y$ calculated by NEKTAR (solid curves) and by DPD (symbols) at $R e=20$ for two different Lagrangian resolutions. The normalized density fields are plotted in the same figures as well. Note that the continuum and the DPD velocity profiles are in excellent agreement for resolution 1 provided the DPD driving force is the continuum pressure gradient over the entire domain. This can be confirmed by the uniform density field obtained for this resolution. The higher particle density results in a smoother density profile specifically at the corners, whereas in 9(c) the particle density is not as smooth, resulting in a loss of accuracy in the vicinity of the cavity. This leads to less accurate results in the vicinity of the groove for resolution 2.

Figures $10(a, b)$ show that the driving force methodology works very well at Re higher than previously attained by use of inflow/outflow conditions, and thus it extends the velocity limit imposed by DPD compressibility. This is not the case when the body force is constant along the channel and zero in the cavity, see Fig. 10(c,d). This result suggests that the continuum pressure gradient over the entire domain is required for DPD simulation to accurately capture the transition 

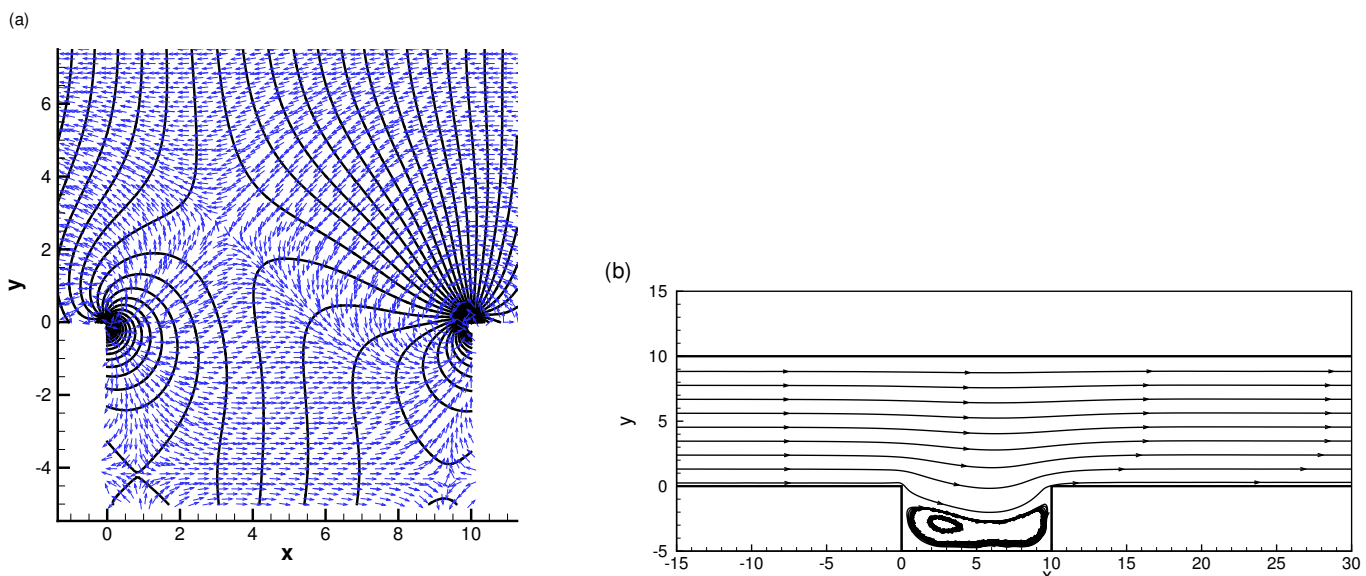

Figure 7: Flow through a channel with rectangular cavity at $R e=20$ : (a) continuum pressure field simulated by NEKTAR, unit vectors indicate pressure gradient direction; (b) streamlines from DPD simulation driven by continuum pressure gradient. Computational channel extends over $x=(-20,50)$.

between the strong channel flow and the much weaker cavity flow including the corner regions. The compressibility effects and the velocity limit in DPD simulations are very well known and have been addressed in several studies before (e.g. $[15,16])$. Table 2 gives the sound speeds for two different DPD resolutions estimated based on the methodology described in [17]. Consequently, we can estimate the maximum Mach numbers in the domain. At $R e=20$, the maximum Mach number for resolution 1 is $\approx 0.49$, whereas this value for resolution 2 is $\approx 0.56$. While the compressibility effects are approximately the same for both resolutions, resolution 1 performs better due to the higher number density as discussed above. Moreover, the use of tailored body forces can mitigate the adverse effects of DPD compressibility. Most notably, at higher $R e=100$ the estimated maximum Mach number is $\approx 2.18$, whereas the average Mach number in the domain is $\approx 1.4$. These values are extremely high for a classic DPD solution to converge as shown in Figs. 10(c,d), whereas the tailored-body-force solution achieves much better results (Figs. 10(a,b)).

Flow in a uniform channel can also be simulated by DPD without a body force driver and instead with the use of inflow-outflow end conditions of Lei et al. ([11]). At $R e=20$ the results so obtained are effectively those of Fig. 8, but at the computational price of about 10 times the number of iterations needed for 
(a)

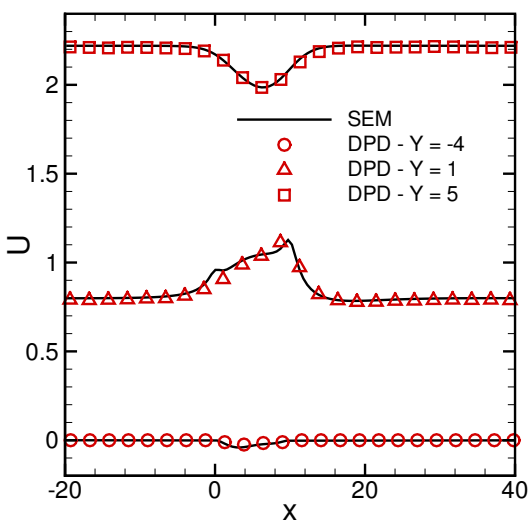

(b)

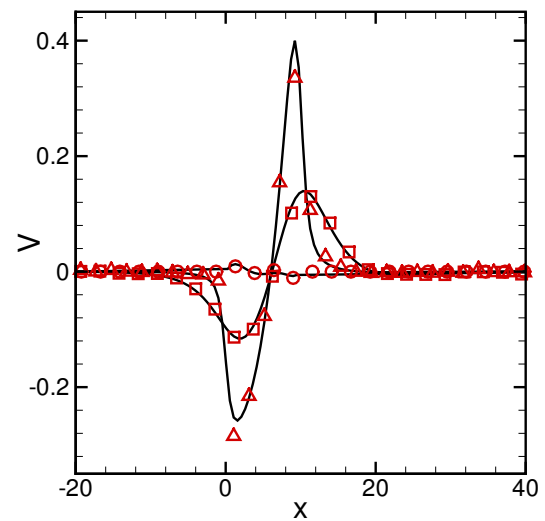

(c)

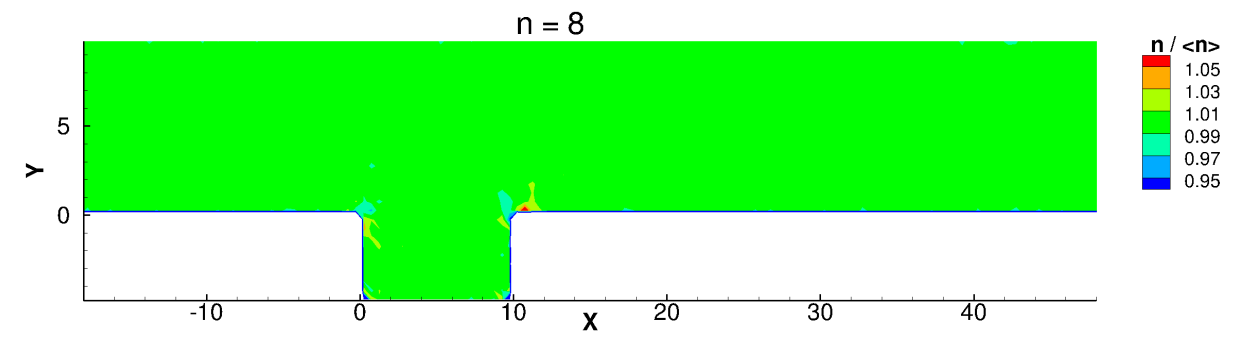

Figure 8: Flow through channel with rectangular cavity at $R e=20$ : (a,b) profiles of $x, y$ - velocity components $U, V$ along lines of constant $y$ in the vicinity of the corners; continuum fields simulated by NEKTAR (SEM); DPD simulation driven by the continuum pressure gradient for resolution 1.

acceptable convergence by the body force driven method. At $R e=100$ no converged solutions were obtained. Lei et al. [11] applied their inflow-outflow end conditions to another irregular channel, the backward-facing step. They found that convergence became more and more difficult as $R e$ increased, and that converged DPD simulations at higher $R e$ were achievable only by increasing the channel gap while holding the bulk velocity constant. This work has demonstrated that body force drivers provide a promising approach to extend DPD simulations to irregular geometries, the restriction to periodic channels not withstanding. The increase in the Reynolds number range, and the efficiency gain due to a reduced number of iterations for convergence are gains measured relative to approaches without body force drivers. 
(a)

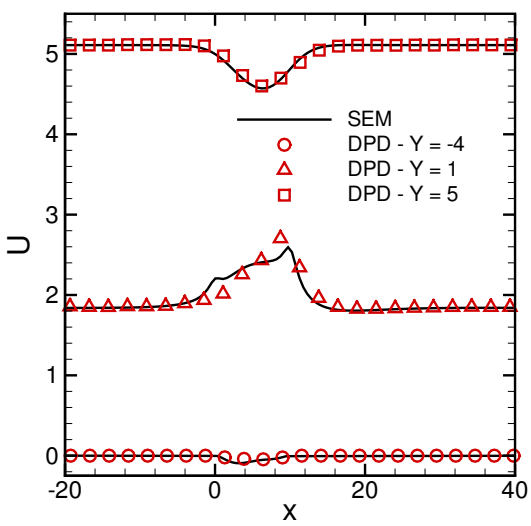

(b)

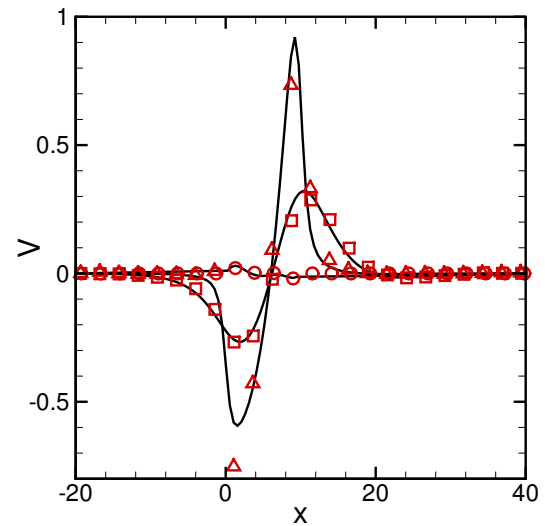

(c)

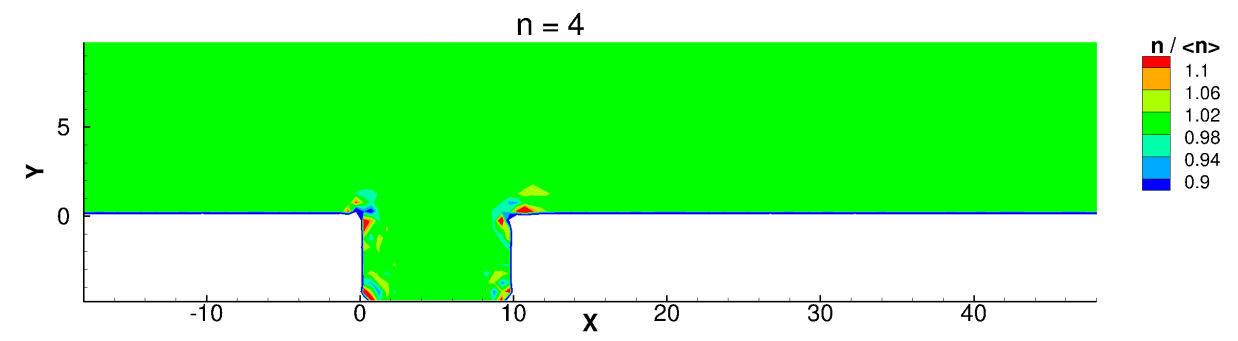

Figure 9: Flow through channel with rectangular cavity at $R e=20$ : (a,b) profiles of $x, y$ - velocity components $U, V$ along lines of constant $y$ in the vicinity of the corners; continuum fields simulated by NEKTAR (SEM); DPD simulation driven by the continuum pressure gradient for resolution 2.

\subsection{Example 3: flow in an asymmetric stenosis}

In order to demonstrate the applicability of the proposed method to more general 3D complex geometries, we have considered a straight tube with length $L=200 \mu m$ with a $75 \%$ asymmetric stenosis. The geometry is plotted in Fig. 11 . The parameters used in the DPD system are given in Table 3 and the maximum inlet velocity is chosen to produce $\operatorname{Re}=V_{\max } D / v=2.5$, where $D$ is the diameter of the tube. We generate the shape of the stenosis using the equations given in [18] with the appropriate parameters to achieve a maximum of $75 \%$ constriction. This non-periodic wall-bounded flow requires the proper imposition of no-slip boundary conditions in the context of DPD [11].

SEM (NEKTAR) was used to solve the N-S equations in the same geometry at $R e=2.5$. The geometry is meshed by tetrahedral elements and Jacobi polynomials of order 5 were used in this simulation. The coupling between the two solvers (SEM and DPD) was carried out by a novel computational interface called 
(a)

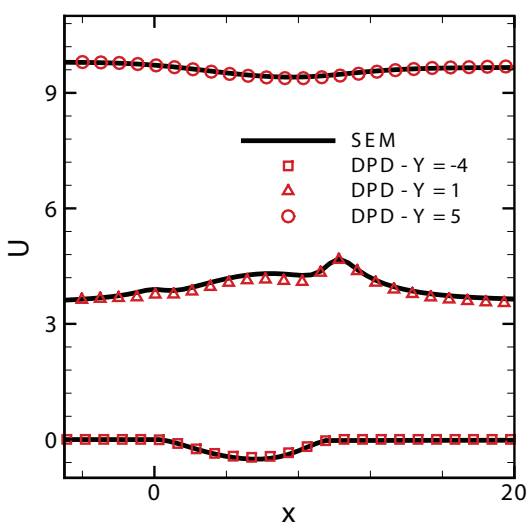

(c)

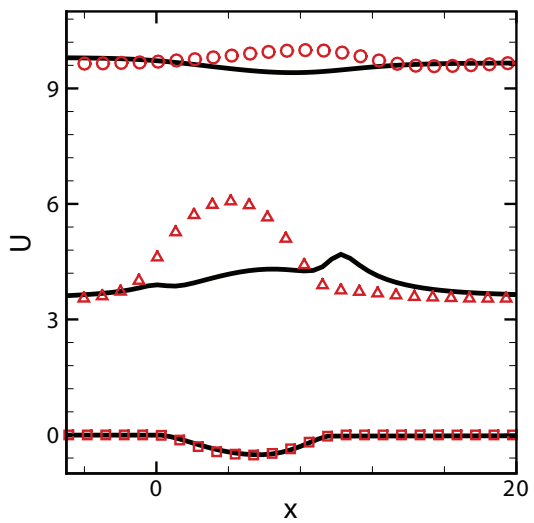

(b)

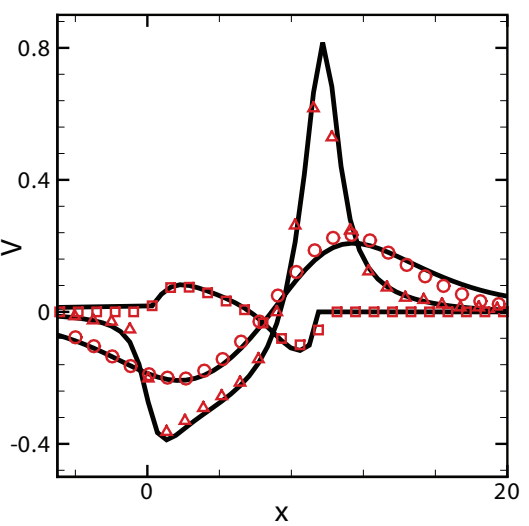

(d)

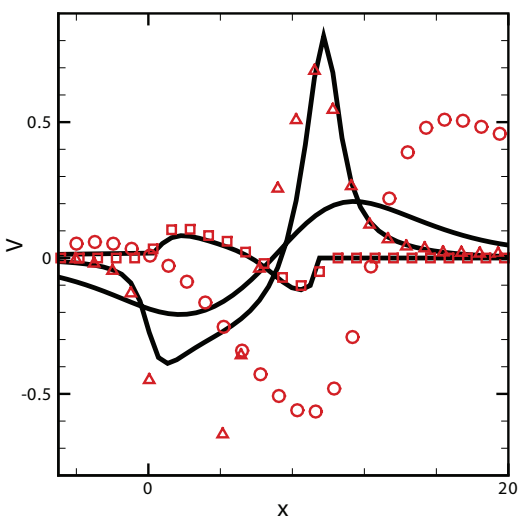

Figure 10: Flow through channel with rectangular cavity at $R e=100$ : profiles of $x-, y-$ velocity components $U, V$ along lines of constant $y$ in the vicinity of the corners: continuum fields (black curves) simulated by NEKTAR; (a,b) DPD simulation (red symbols) driven by the continuum pressure gradient over the entire domain; (c,d) DPD simulation driven by a constant body force along the channel and the cavity.

Multiscale Universal Interface (MUI) [19]. MUI works as a storage buffer between different heterogenous solvers. Data are passed between solvers at discrete collocation points regardless of the type of grid or framework (Eulerian vs. Lagrangian), and an interpolation is performed using a Gaussian kernel to interpolate the pressure gradients at the location of particles; for more details on MUI we refer the readers to [19]. 


\begin{tabular}{ccccccccc}
\hline & $n$ & $a_{c}$ & $a_{d}$ & $s$ & $r_{c}$ & $k_{B} T$ & $v$ & $c_{s}$ \\
\hline example3 & 4.0 & 18.75 & 4.5 & 0.205 & 1.58 & 1.0 & 6.62 & 10.3
\end{tabular}

Table 3: DPD parameters: same parameters as in Table 1.

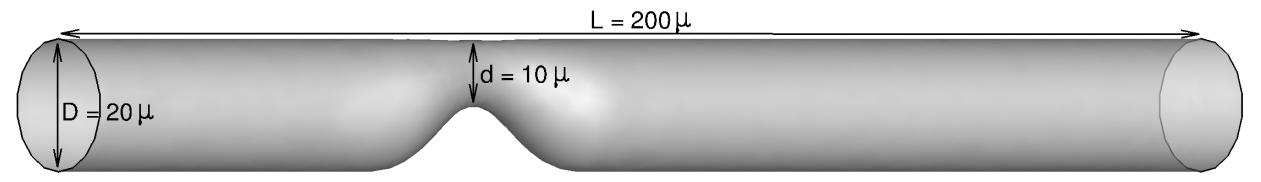

Figure 11: The schematic of the 3D irregular geometry and its dimensions used in this example. The bump in the tube represents a stenosis occluding $75 \%$ of the tube at its apex.

We first use a constant body-force to drive the DPD fluid, and the resulting particle density contours are shown in Fig. 12(a). Contours are plotted on a midplane slice $(Z=0)$ of the tube. It is clear that the constriction leads to accumulation of particles upstream of stenosis. This will yield a completely wrong velocity profile throughout the tube (results not shown here). Figure 12(b) shows the contours of density for the same flow driven by a properly scaled pressure-gradient calculated by SEM. The contours show more uniform particle densities in the constriction leading to significantly improved velocity field in the domain. We present contours of axial velocity $U$ in Fig. 12(c). The maximum velocity occurs at the throat of the stenosis and is $\approx 3.12$. Based on the sound speed from Table 3 , and assuming a uniform density throughout the domain (Fig. 12(b)), the average and maximum Mach numbers can be estimated as $\approx 0.07$ and 0.3 , respectively, which may cause slight compressibility effects at the constriction. However, interestingly, the tailored body-force can alleviate this issue by correcting the density field in the domain.

In order to have a closer look at the axial $U$ and transversal velocities $V$, we extract the velocities on $Z=0$ plane at $r=0$ and $r=4$ along the tube length. Figure 13(a,b) show the results for the axial and transversal velocities respectively. Very good agreement can be achieved for this flow with the use of a tailored body force. 
(a)

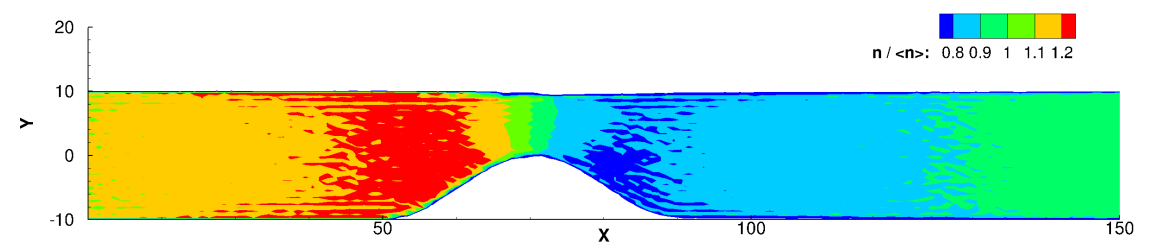

(b)

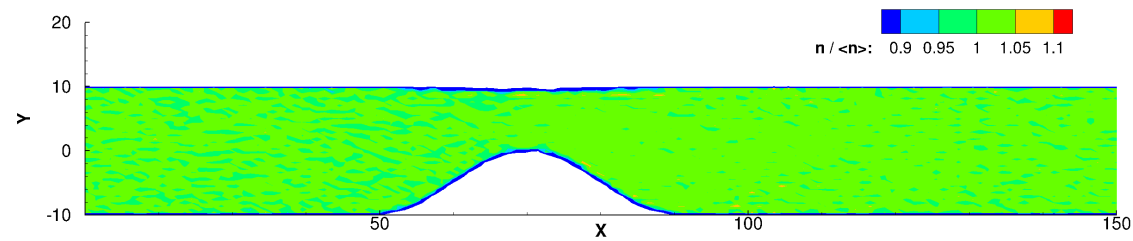

(c)

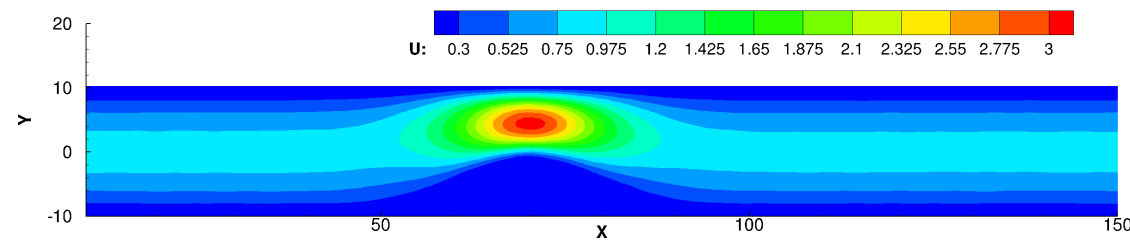

Figure 12: DPD results of flow simulation in a constricted tube at $R e=2.5$. Contours of density and velocity on the mid-plane $(Z=0)$ : (a) density contours for the constant-body-force driven flow; (b) density contours when the geometry-aware body-force is used; (c) axial velocity contours for the case in (b).

(a)

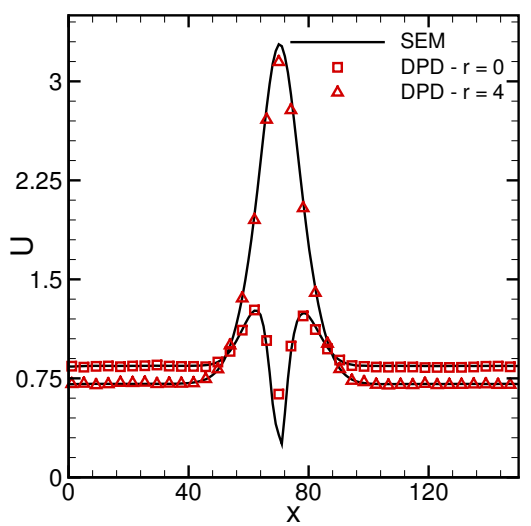

(b)

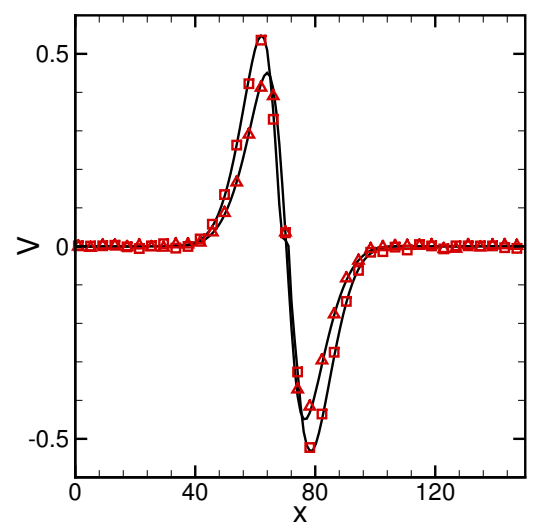

Figure 13: Flow through stenosis at $R e=2.5$ : (a,b) profiles of $r, \theta$ - velocity components $U, V$ along lines of constant $y$; continuum fields simulated by NEKTAR (SEM); DPD simulation driven by the continuum pressure gradient for the same $R e$. 


\section{Conclusion}

The hypothesis tested in this work with three examples is that the DPD simulations of flows in complex or irregular geometries are more easily carried out when the particles are subjected to a suitably chosen body force field. The two and three-dimensional examples presented here have demonstrated that particle driving forces derived as the gradient of the Navier-Stokes pressure field faithfully reproduce the continuum flow field obtained either analytically or numerically. We need to emphasize that while the current approach has been tested for steadystate problems, there is no limitation on the use of this methodology for unsteady problems. Transient flows have been modeled by DPD [20], and time-dependent body forces have been employed in DPD simulations of Poiseuille flow before (for example, the use of Womersely pressure-driven flow in [11]). The major restriction for transient problems is imposed by the finite compressibility effects of DPD fluid, which will set an upper limit for the frequency of the transient dynamics.

The purpose of the body-force method is to model complex-fluid flows such as suspension flow in irregular geometries. The concept of body-force drivers to enhance particle methods is straightforward; implementation requires a choice of a force field, which in this work is the pressure gradient derived from the continuum $\mathrm{N}-\mathrm{S}$ solution. This choice is motivated by the availability of a variety of numerical $\mathrm{N}-\mathrm{S}$ solvers, which yield the required fields. In this work the first task has been to demonstrate that the DPD simulation employing the N-S pressure gradient yields the original N-S flow, an apparently zero-sum game. However, in Example 2 it was shown that a crude approximation of the N-S pressure gradient yields a less accurate DPD simulation. The plots below shown how the use of the N-S pressure gradient can be applied to drive the flows of complex fluids, such as suspensions of red blood cells and platelets in a Newtonian plasma. Fig. 14(a) presents a snapshot of this blood suspension flowing in a microchannel with stenosis, while Fig. 14(b) displays contours of the characteristic plug-like streamwise velocity profiles

in the center plane of the channel. In the next example the driving force field of example 1 is employed to study the dynamics of an inextensible bead-spring chain inserted into the stagnation region. Fig. 15 shows the instantaneous streamlines and velocity vectors of the now disturbed stagnation-point flow of Fig. 3. The disturbance of the stagnation-point flow is ignored in Brownian dynamics, and this simulation appears to be the first time it has been captured and visualized.

DPD simulations of flow in irregular geometries poses challenges mainly due to local accumulation or depletion of DPD particles, which leads to undesirable nonuniform particle density throughout the domain. This, in turn, affects the local 
DPD fluid properties such as viscosity and sound speed leading to moderate to severe numerical errors. The proposed methodology along with the numerical experiments in this paper have shown that a tailored body-force can effectively remove the artifacts yielding a significantly improved particle density in the domain. In summary, the results given above suggest that the advantages of the force driven scheme relative to other methods are:

- efficiency gains measured by the number of iterations required for convergence.

- convergence at higher flow rates (Reynolds number) where other methods fail.

while the limitations are:

- not applicable for the problems with moving interfaces.

\section{Acknowledgment}

This work was sponsored by the Collaboratory on Mathematics for Mesoscopic modeling of Materials (CM4) supported by DOE, and also by NIH grant (U01HL114476). Computations were performed using a DOE INCITE award and a NSF XSEDE award (TGDMS140007). AY would like to thank Dr. Xin Bian for his assistance with providing data for the sound speeds, and Yuhang Tang for his guidance and notes regarding MUI. 


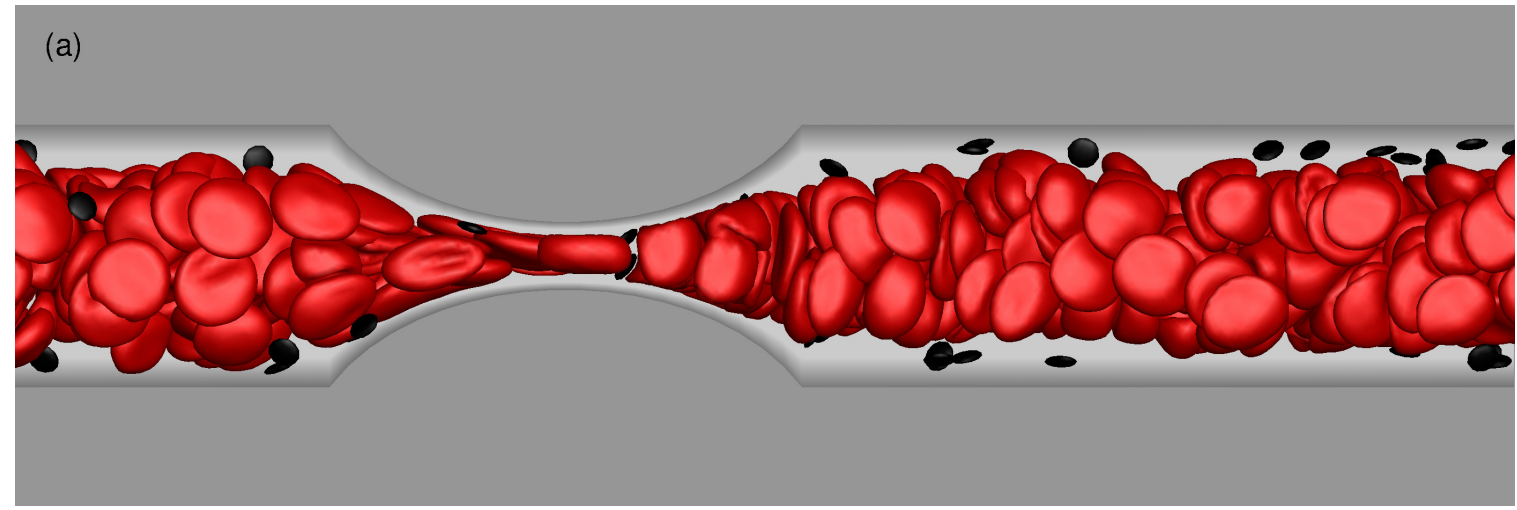

(b)
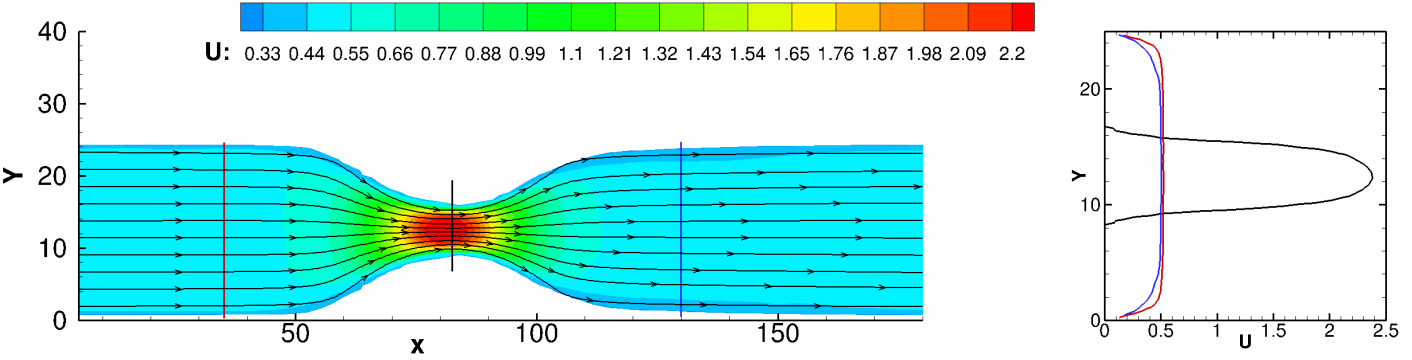

Figure 14: (a) Snapshot of the whole-blood simulation in a micro-channel device with $75 \%$ degree stenosis. The cross-sectional area of the channel is a square of $25 \times 25$ micron, and blood is at $35 \%$ hematocrit. Red cells represent the deformable red blood cells and black ellipsoidal cells are non-deformable platelets in their resting form. The driving body force is the non-uniform pressure gradient derived from SEM solution of Navier-Stokes equation at $\mathrm{Re}=0.1$ for the same geometry. (b) left: Velocity contours and streamlines for the flow of blood suspension in the 75\%-stenosis microchannel; right: profiles of streamwise velocity component at three different locations along the microchannel length specified by the same colors. A plug-like velocity profile is achieved for the blood suspension.

\section{Appendix}

Potential Flow: The numbering in Fig. 1 is designed to accurately approximate the potential within a periodic square with center $z=0$. Each diagonal vortex row is numbered with indices $(j, k)$ of its origin (the comma is omitted in Fig. 1); the label $j=1,2,3, \ldots$ stands for successive polygons enclosing the center (red circle), and odd/even values of integer $k$, respectively, denote the negative/positive vortices located at the corners of polygon $j$. Odd/even $j$ indicate squares/octagons whose perimeters lie ever further from $z=0$ as $j$ increases. Thus, in Fig. 1 negative vortices $(1,1)$ and $(1,3)$ are origins of rows passing through the corners of the periodic square, while positive vortices $(2,2)$ and $(2,4)$ are origins of rows passing 


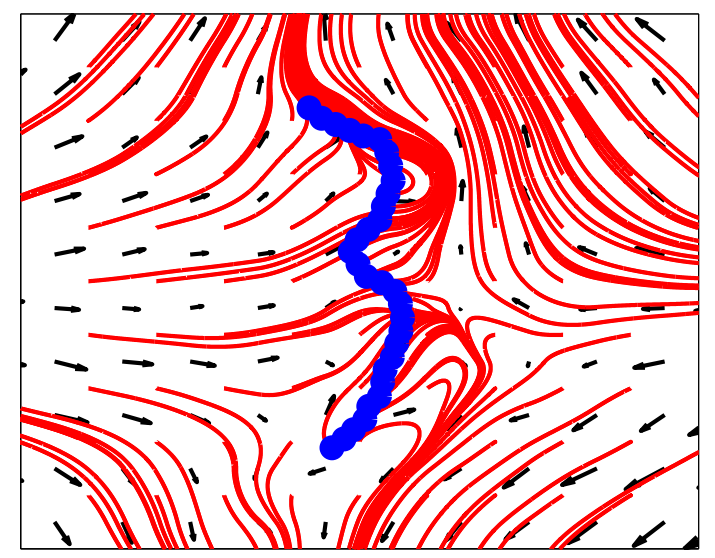

Figure 15: Stagnation-type flow: Instantaneous streamlines and velocity vectors showing the disturbance of the stagnation-point flow caused by the inextensible filament constrained to deform in the plane. The driving body force is the pressure gradient of the potential flow presented in section 3.1.

though octagon $j=2$.

The complete potential for the lattice of vortices is constructed as a weighted sum of the row potentials (11) for the rows passing through the numbered vortices in Fig. 1. The potential is constructed in stages with terms $W_{j}$ being the contributions from polygon $j$ enclosing the center (red circle) of Fig. 1 . The square $j=1$ accounts for the rows passing diagonally in each direction through its corner vortices, and by their proximity to the center these constitute the main contribution inside that square. Along the right diagonal the positive vortices $(1,2),(1,4)$ have $A=b=A_{r}$ and along their left diagonal $A=A_{l}, b=A_{r}$, and likewise along the left diagonal the negative vortices $(1,1),(1,3)$ have $A=b=A_{l}$ and along their right diagonal $A=A_{r}, b=A_{l}$. The real $n$ and imaginary $m$ parts of the ratio $b / A$ for these are given in the first four rows of Table 4 , and with these values the first approximation to the potential is constructed from equations $(12,11)$ as:

$$
2 W_{1}=W_{c}\left(z *_{r}, 0\right) \mp W_{c}\left(z *_{l}, 0\right)+W_{s}\left(z *_{l}, 1\right) \mp W_{s}\left(z *_{r}, 1\right) .
$$

The derivatives $d W_{1} / d z$ and $d W_{1}^{2} / d z^{2}$ are constructed analogously. Because the diagonal rows in each direction pass through the corner vortices of the square $j=1$ a weight of $1 / 2$ is applied to $W_{1}$. The negative/positive signs indicate the stagnation-point/circulation flows respectively, and all extraneous constants 


\begin{tabular}{cccccc}
$j$ & $k$ & $A$ & $b$ & $n$ & $m$ \\
\hline 1,1 & 1,3 & $A_{l}$ & $A_{l}$ & 1 & 0 \\
1,2 & 1,4 & $A_{r}$ & $A_{r}$ & 1 & 0 \\
1,1 & 1,3 & $A_{r}$ & $A_{l}$ & 0 & 1 \\
1,2 & 1,4 & $A_{l}$ & $A_{r}$ & 0 & -1 \\
2,1 & 2,7 & $A_{l}$ & $A_{r}+i 2 a$ & 1 & -2 \\
2,2 & 2,8 & $A_{r}$ & $A_{l}+i 2 a$ & 1 & 2 \\
2,3 & 2,5 & $A_{l}$ & $A_{r}+2 a$ & -1 & -2 \\
2,4 & 2,6 & $A_{r}$ & $A_{l}-2 a$ & -1 & 2 \\
3,3 & 3,9 & $A_{r}$ & $3 A_{l}$ & 0 & 3 \\
3,4 & 3,10 & $A_{l}$ & $3 A_{r}$ & 0 & -3 \\
4,1 & 4,7 & $A_{l}$ & $3 A_{r}+i 2 a$ & 1 & -4 \\
4,2 & 4,8 & $A_{r}$ & $3 A_{l}+i 2 a$ & 1 & 4 \\
4,3 & 4,5 & $A_{l}$ & $3 A_{r}+2 a$ & -1 & -4 \\
4,4 & 4,6 & $A_{r}$ & $3 A_{l}-2 a$ & -1 & 4
\end{tabular}

Table 4: Real $n$ and imaginary $m$ parts of the complex constant $b / A$ for the sums of row potentials, equations $(11,12)$, for pairs of vortex rows having origins $j$ and $k$ corresponding to the vortex numbering of Fig. 1.

are omitted. The strength $\kappa /(4 \pi)$ is applied when all terms are completed. The second approximation $W_{2}$ is constructed from pairs of vortices located on octagon $j=2$; these have already been added with a $1 / 2$ weight along the rows counted in the construction of $W_{1}$. Now they are added with a $1 / 2$ weight along diagonals not previously traversed. The positive pairs $(2,2),(2,8)$ and $(2,4),(2,6)$ are connected by lines through the origin $z=0$ with $A=A_{r}$, and $b=a+i 3 a$, $b=3 a+i a$ respectively. Likewise, the negative pairs $(2,1)(2,7)$ and $(2,3),(2,5)$ have $A=A_{l}$, and $b=a+i 3 a, b=3 a+i a$ respectively. The third approximation $W_{3}$ is similarly obtained. From their $n, m$ values listed in Table 4 the second, third and fourth approximations are constructed from equations (11):

$$
2 W_{2}=W_{c}\left(z *_{r}, 2\right) \mp W_{c}\left(z *_{l}, 2\right), 2 W_{3}=W_{s}\left(z *_{l}, 3\right) \mp W_{s}\left(z *_{r}, 3\right),
$$

and

$$
2 W_{4}=W_{c}\left(z *_{r}, 4\right) \mp W_{c}\left(z *_{l}, 4\right) .
$$

The total potential $W=-i \kappa /(2 \pi)\left(W_{1}+W_{2}+W_{3}+W_{4} \ldots\right)$, equation(14), can be inferred from these $W_{j}$ by induction with the aid of figure(1) by continuing the 
pattern of squares and octagons displayed in Table 4. As the perimeter of polygon $j$ lies ever further from the center $z=0$ the denominator of $W_{j}$ increases rapidly. This becomes evident when the term of type $\sin ^{2}(\pi z / A)$ is written in detail. Hence, the potential and its derivatives in any periodic square can be represented accurately with only a few terms.

Dissipative Particle Dynamics (DPD) Method: In the standard DPD method, the particles interact via pairwise forces, consisting (in the basic form) of three components: (i) conservative force $\mathbf{f}^{C}$; (ii) dissipative force, $\mathbf{f}^{D}$; and (iii) random force, $\mathbf{f}^{R}$. Hence, the total force on particle $i$ is given by $\mathbf{f}_{i}=\sum_{i \neq j}\left(\mathbf{f}_{i j}^{C}+\mathbf{f}_{i j}^{D}+\mathbf{f}_{i j}^{R}\right)$, where the sum acts over all particles within a cut-off radius $r_{c}$. Specifically,

$$
\mathbf{f}_{i}=\sum_{i \neq j} a_{c} \omega\left(r_{i j}\right) \hat{\mathbf{r}}_{i j}-a_{d} \omega^{2}\left(r_{i j}\right)\left(\hat{\mathbf{r}}_{i j} \cdot \hat{\mathbf{v}}_{i j}\right) \hat{\mathbf{r}}_{i j}+\sigma \omega\left(r_{i j}\right) \frac{\zeta_{i j}}{\sqrt{\Delta t}} \hat{\mathbf{r}}_{i j}
$$

where $a_{c}$ is a maximum repulsion between DPD particles, $r_{i j}$ is the distance with the corresponding unit vector $\hat{\mathbf{r}}_{i j}, \hat{\mathbf{v}}_{i j}$ is the difference between the two velocities, $\zeta_{i j}$ is a Gaussian random number with zero mean and unit variance, and $a_{d}$ and $\sigma$ are parameters coupled by $\sigma^{2}=2 a_{d} k_{B} T$. Typically, the weighting functions $\omega\left(r_{i j}\right)$ are given by

$$
\omega\left(r_{i j}\right)= \begin{cases}\left(1-\frac{r_{i j}}{r_{c}}\right)^{S} & r_{i j}<r_{c} \\ 0 & r_{i j} \geq r_{c}\end{cases}
$$

More details on the basic formulation of the DPD method can be found in $[21,22]$.

\section{References}

[1] H. Lei, D. Fedosov, B. Caswell, G. Karniadakis, Blood flow in small tubes: Quantifying the transition to the non-Newtonian regime, J. Fluid Mech. 722 (2013) 214-239.

[2] S. Chen, N. Phan-Thien, B. C. Khoo, X. J. Fan, Flow around spheres by dissipative particle dynamics, Physics of Fluids (1994-present) 18 (10) (2006) 103605.

[3] X. Fan, N. Phan-Thien, S. Chen, X. Wu, T. Y. Ng, Simulating flow of DNA suspension using dissipative particle dynamics, Physics of Fluids (1994present) 18 (6) (2006) 063102. 
[4] X. Li, A. Popel, G. E. Karniadakis, Blood-plasma separation in Y-shaped bifurcating microfluidic channels: a dissipative particle dynamics simulation study, Phys. Biol. 9 (2012) 026010.

[5] G. E. Karniadakis, S. Sherwin, Spectral/hp Element Methods for Computational Fluid Dynamics, Oxford University Press, Oxford, 2 edn., 2005.

[6] R. R. Lagnado, L. G. Leal, The visualization of three-dimensional flow in a four-roll mill, Experiments in Fluids 9 (1990) 25-32.

[7] H. Yang, C. C. Park, Y. T. Hu, , L. G. Leal, The coalescence of two equalsized drops in a two-dimensional linear flow, Phys. Fluids 13 (2001) 1087.

[8] J. Deschamps, V. Kantsler, E. Segre, V. Steinberg, Dynamics of a vesicle in general flow, Proceedings of the National Academy of Sciences 106 (28) (2009) 11444-11447.

[9] L. M. Milne-Thomson, Theoretical Hydrodynamics, The Macmillan Company, New York, 4 edn., 1960.

[10] H. B. Dwight, Tables of Itegrals and Other Mathematical Data, The Macmillan Company, New York, 4 edn., 1961.

[11] H. Lei, D. A. Fedosov, G. E. Karniadakis, Time-dependent and outflow boundary conditions for Dissipative Particle Dynamics, J.Comp. Phys. 230 (2011) 3765-3779.

[12] J. A. Backer, C. P. Lowe, H. C. J. Hoefsloot, P. D. Iedema, Poiseuille flow to measure the viscosity of particle model fluids, J. Chem. Phys. 122 (2005) 154503.

[13] M. Deng, L. Grinberg, B. Caswell, G. E. Karniadakis, Effects of thermal noise on the transitional dynamics of an inextensible elastic filament in stagnation flow, Soft Matter.

[14] D. Pan, N. Phan-Thien, N. Mai-Duya, B. C. Khoo, Numerical investigations on the compressibility of a DPD fluid, J.Comp. Phys. 242 (2013) 196-210.

[15] D. A. Fedosov, I. V. Pivkin, G. E. Karniadakis, Velocity limit in DPD simulations of wall-bounded flows, Journal of Computational Physics 227 (4) (2008) 2540-2559. 
[16] D. Pan, N. Phan-Thien, N. Mai-Duy, B. C. Khoo, Numerical investigations on the compressibility of a DPD fluid, Journal of Computational Physics 242 (2013) 196-210.

[17] X. Bian, Z. Li, M. Deng, G. E. Karniadakis, Fluctuating hydrodynamics in periodic domains and heterogeneous adjacent multidomains: Thermal equilibrium, Physical Review E (to appear) .

[18] M. Deshpande, D. Giddens, R. Mabon, Steady laminar flow through modelled vascular stenoses, Journal of Biomechanics 9 (4) (1976) 165-174.

[19] Y.-H. Tang, S. Kudo, X. Bian, Z. Li, G. E. Karniadakis, Multiscale Universal Interface: A concurrent framework for coupling heterogeneous solvers, Journal of Computational Physics 297 (2015) 13-31.

[20] I. V. Pivkin, G. E. Karniadakis, A new method to impose no-slip boundary conditions in dissipative particle dynamics, Journal of Computational Physics 207 (1) (2005) 114-128.

[21] R. D. Groot, P. B. Warren, Dissipative particle dynamics: Bridging the gap between atomistic and mesoscopic simulation, Journal of Chemical Physics 107 (11) (1997) 4423.

[22] P. Espanol, P. Warren, Statistical mechanics of dissipative particle dynamics, EPL (Europhysics Letters) 30 (4) (1995) 191. 